\title{
Mathematic Identifiability
}

National Cancer Institute

\section{Source}

National Cancer Institute. Mathematic Identifiability. NCI Thesaurus. Code C94927.

Ability to achieve reasonable accuracy and precision in determining a particular parameter when fitting a mathematical model to data. 